\title{
Muon g-2 and Hadronic Vacuum Polarization: Recent Developments
}

\author{
Simon Eidelman ${ }^{1,2, a}$ \\ ${ }^{1}$ Budker Institute of Nuclear Physics, Novosibirsk 630090, Russia \\ ${ }^{2}$ Novosibirsk State University, Novosibirsk 630090, Russia
}

\begin{abstract}
We discuss various experiments on $e^{+} e^{-}$annihilation into hadrons relevant to the problem of the muon anomalous magnetic moment. They include a status of the ISR measurements of the $e^{+} e^{-} \rightarrow \pi^{+} \pi^{-}$as well as studies of numerous hadronic final states in experiments with the CMD-3 and SND detectors at the VEPP-2000 $e^{+} e^{-}$collider.
\end{abstract}

\section{Introduction}

The anomalous magnetic moment of the muon is one of the most precisely known physical quantities. In 2006 the BNL E821 Collaboration published the final results of their measurement of the muon $a_{\mu} \equiv\left(g_{\mu}-2\right) / 2$ [1]. Various calculations show that the Standard Model (SM) prediction is about 3.5 standard deviations below the experimental value $[2,3]$. At the moment two new measurements of $a_{\mu}$, each aimed at four-fold increase of accuracy, are planned at Fermilab and J-PARC. If the central value of the experimental result is confirmed, the deviation between experiment and theory will reach 8-10 standard deviations unambiguously pointing to effects of $\mathrm{New}$ Physics.

The theoretical prediction accuracy is currently limited by the uncertainties of the hadronic vacuum polarization extracted from the cross sections of $e^{+} e^{-}$annihilation into hadrons measured by a scan method at CMD-2 and SND detectors at the VEPP-2M and initial-state radiation (ISR) at BaBar (Fig. 1), see the review of $e^{+} e^{-}$experiments in Ref. [4].

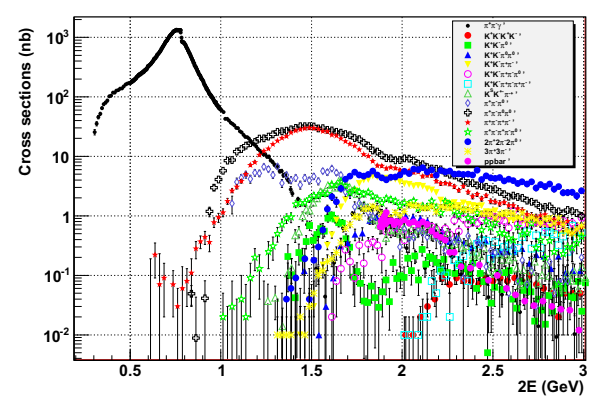

Figure 1. Current status of exclusive measurements [4]
As it is absolutely necessary to improve experimental accuracy, experiments on low-energy $e^{+} e^{-}$annihilation into hadrons are currently in progress in various centers.

In addition to the low-energy measurements, other energy ranges are still of interest for $a_{\mu}$ and particularly for the $\alpha\left(M_{Z}^{2}\right)$ determination. Recently a new measurement of $R$ was performed between 3.12 and $3.72 \mathrm{GeV}$ in Novosibirsk using the KEDR detector [5]. The achieved systematic uncertainty is $2.1 \%$ with a total uncertainty of $3.3 \%$. The results are shown in Fig. 2. Analysis of the KEDR

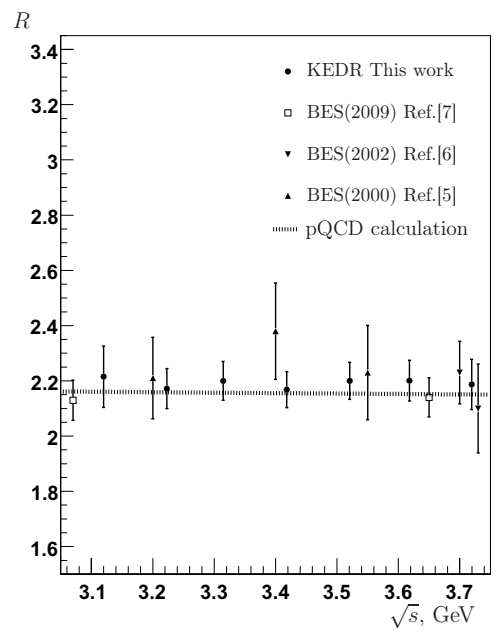

Figure 2. $R$ measurement at KEDR [5]

scan between $1.9 \mathrm{GeV}$ and $J / \psi[6]$ is in progress while $\mathrm{R}$ measurements from 2 to $4.6 \mathrm{GeV}$ with BESIII and further studies of the charmonium region with KEDR are also planned. Improvement to about $2 \%$ in total can be expected from joint efforts of BESIII and KEDR.

\footnotetext{
ae-mail: simon.eidelman@cern.ch
} 


\section{ISR measurements of $e^{+} e^{-} \rightarrow \pi^{+} \pi^{-}$}

The process $e^{+} e^{-} \rightarrow \pi^{+} \pi^{-}$is known to give the largest contribution to the leading-order hadronic term $a_{\mu}^{\mathrm{LO} \text {,had }}$ about $73 \%$. Recent ISR measurements of this process substantially improved the accuracy of its cross section. The BaBar Collaboration used a data sample collected at the peak of the $\Upsilon(4 S)$ resonance to achieve the record precision of about $0.5 \%$ near the $\rho$ meson peak $[7,8]$.

KLOE was using ISR running at the $\phi$ meson peak and gradually increased the precision of their measurements to $0.7 \%$ [9-11]. However, the results of KLOE and BaBar differ with the discrepancy reaching $5 \%$ in some energy regions, far beyond the declared precision.

A very recent precise measurement has been performed by the BES Collaboration running at the $\psi(3770)$ peak [12]. Their ISR measurement reached precision of about $0.9 \%$, see the cross section in Fig. 3 .

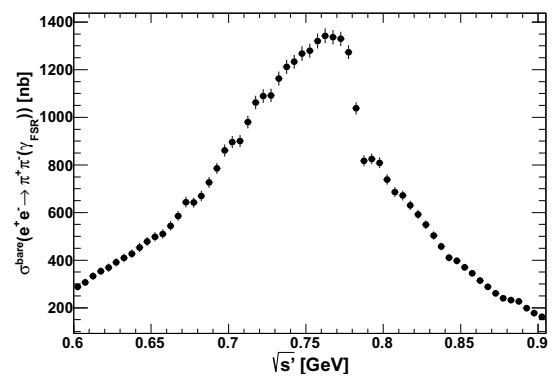

Figure 3. Cross section of $e^{+} e^{-} \rightarrow \pi^{+} \pi^{-}(\gamma)$ at BESIII [12]

Comparison between the results of BESIII and those from other groups - SND [13], CMD-2 [14], BaBar and KLOE shows that there are local discrepancies between the data of BaBar, KLOE and BESIII. This can be seen from Figure 4, which illustrates how the results obtained by ISR look like in terms of the hadronic contribution to muon anomaly after integration. The BESIII result lies between those of BaBar and KLOE being somewhat closer to the latter. Obviously, new measurements with comparable or even better precision are needed.

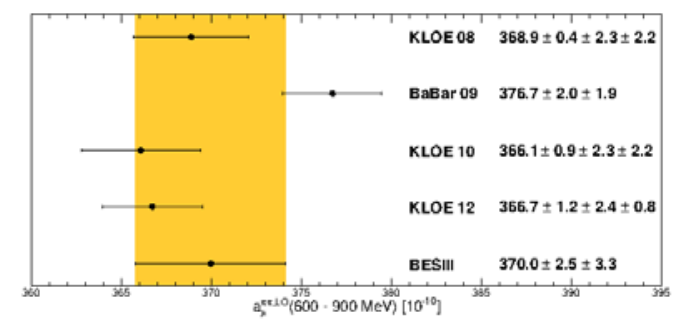

Figure 4. Comparison of BESIII results on $a_{\mu}$ [12]

\section{Experiments at VEPP-2000}

In 2010 a new low-energy $e^{+} e^{-}$collider, VEPP-2000, was commissioned in Novosibirsk [15]. In 2011 data taking started with two detectors, CMD-3 and SND. An integrated luminosity of $\sim 60 \mathrm{fb}^{-1}$ was collected by each in 2011 - 2013 in the center-of-mass (c.m.) energy range 320 - $2000 \mathrm{MeV}$. Figure 5 illustrates accumulation of luminosity in various energy ranges. Lately analysis has been mainly focused on the c.m. energies above the $\phi$ meson. In particular, both groups made an attempt to improve the precision of various cross sections important for the muon anomaly problem [16].

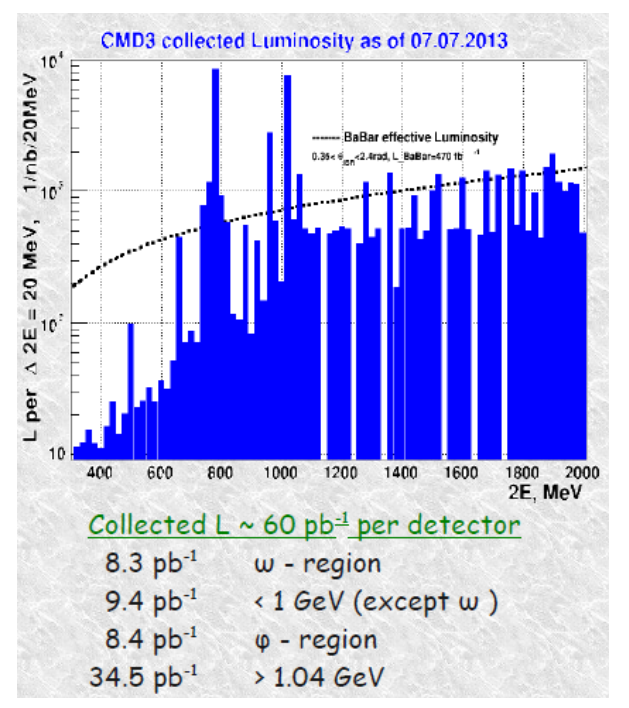

Figure 5. Luminosity collected at VEPP-2000

Measurements of an integrated luminosity at CMD-3 use two processes, $e^{+} e^{-} \rightarrow e^{+} e^{-}$and $e^{+} e^{-} \rightarrow \gamma \gamma$, allowing a precision of $\sim 1 \%$ [17]. At SND, events of large-angle Bhabha scattering are used to determine an integrated luminosity with a systematic accuracy of $2 \%$ [18].

CMD-3 declares an aggressive goal of reaching $0.35 \%$ accuracy in the measurement of the pion form factor, see their prelimiary results in Fig. 6 [19]. Up to to the c.m.

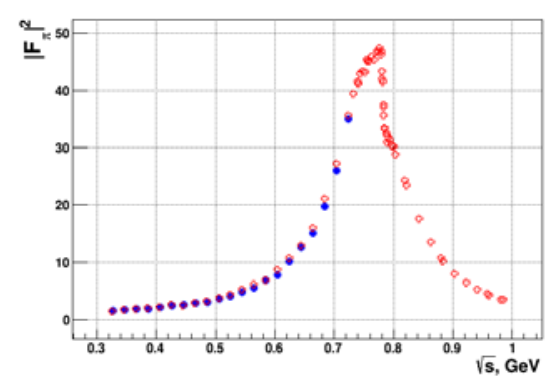

Figure 6. CMD-3 results on the pion form factor

energy of $700 \mathrm{MeV}$ they can separate pions from muons by two independent methods - from the information from the drift chamber and from the electromagnetic calorimeters, the latter being used in the whole energy range.

Both detectors have continued analysis of the collected data samples and are reporting measurements of cross sections for various processes with pions and $\eta$ mesons. SND 

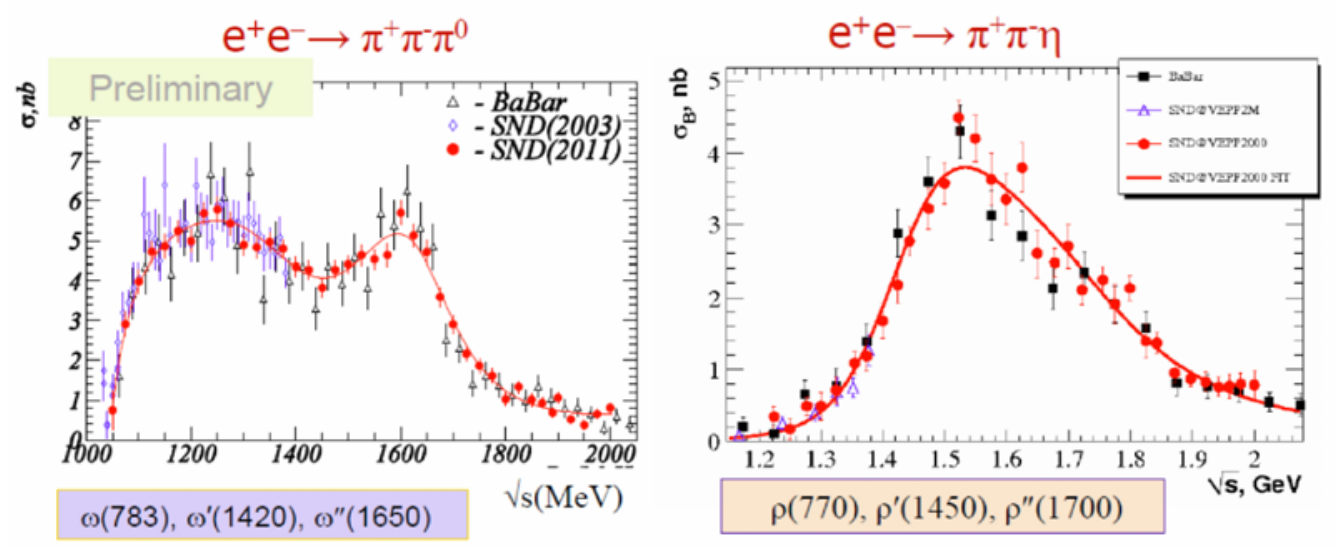

Figure 7. Recent results on hadronic cross sections from SND: (left) $e^{+} e^{-} \rightarrow \pi^{+} \pi^{-} \pi^{0}$ [20], (right) $e^{+} e^{-} \rightarrow \pi^{+} \pi^{-} \eta[18]$

published their final results on $e^{+} e^{-} \rightarrow \pi^{+} \pi^{-} \pi^{0}$ [20], Fig. 7 (left). and $e^{+} e^{-} \rightarrow \pi^{+} \pi^{-} \eta$ [18], Fig. 7 (right).

The cross section of the process $e^{+} e^{-} \rightarrow \pi^{+} \pi^{-} \pi^{0}$ clearly shows two excitations of the $\omega(783)$ meson $\omega(1420)$ and $\omega(1650)$. On the contrary, the energy dependence of the process $e^{+} e^{-} \rightarrow \pi^{+} \pi^{-} \eta$ is dominated by the $\rho(770)$ recurrencies $-\rho(1450)$ and $\rho(1700)$. In both cases the SND data from VEPP-2000 are consistent with the results from VEPP-2M and BaBar and have comparable precision. CMD-3 has also measured the cross sections of these processes with comparable precision, not shown here.

CMD-3 and SND studied all three possible charge combinations of the six-pion production. The cross section in Fig. 8. shows the case when all pions are charged with the obvious dip around the $N \bar{N}$ threshold [21].

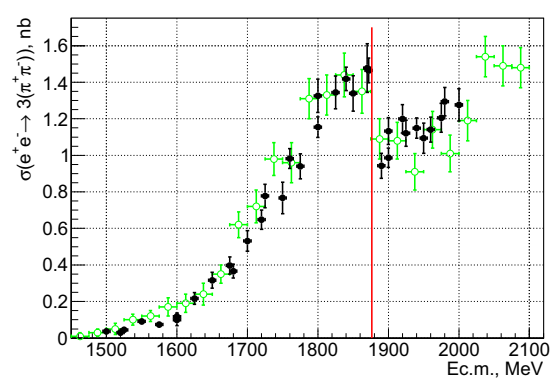

Figure 8. Cross section of $e^{+} e^{-} \rightarrow 3 \pi^{+} 3 \pi^{-}$at CMD-3 [21]

Such behavior of the cross section has been observed before at BaBar [22] as well as in much earlier $e^{+} e^{-}$ and photoproduction measurements, therefore suggesting a probable threshold effect due to the opening of the $N \bar{N}$ channel [23]. Near the $N \bar{N}$ threshold the behavior of the cross section for the $2 \pi^{+} 2 \pi^{-} 2 \pi^{0}$ final state (see Fig. 9) is also irregular, but somewhat differs from that in the $3 \pi^{+} 3 \pi^{-}$case [24].

Finally, SND has measured for the first time ever the cross section of $e^{+} e^{-} \rightarrow \pi^{+} \pi^{-} 4 \pi^{0}$, where the size of the data sample does not allow any conclusions about the $N \bar{N}$ threshold, Fig. 10. These final states have interesting

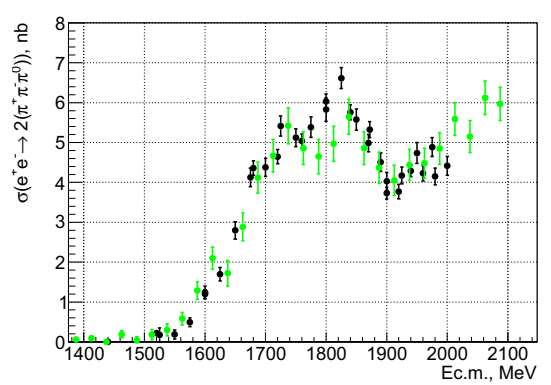

Figure 9. Cross section of $e^{+} e^{-} \rightarrow 2 \pi^{+} 2 \pi^{-} 2 \pi^{0}$ at CMD-3

and rich dynamics which analysis should be performed simultaneously for all three final states. For example, the $\rho f_{0}(1370)$ intermediate mechanism can result in all three possible charge combinations of the six-pion state (the fully neutral $6 \pi^{0}$ state can not be produced in one-photon annihilation because of $C$-parity violation). There are also mechanisms which do not give all charged final states, e.g., $\omega 3 \pi$ or $\eta 3 \pi$. An additional problem is that such final states can have negative $G$-parity whereas normally sixpion states should have positive $G$-parity.

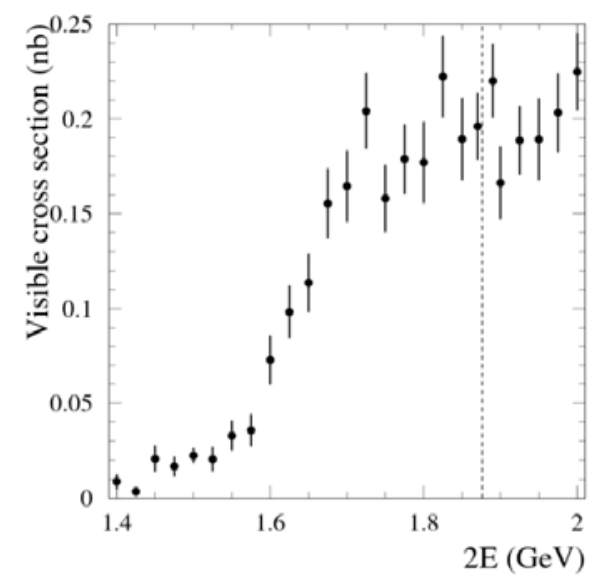

Figure 10. Visible cross section of $e^{+} e^{-} \rightarrow \pi^{+} \pi^{-} 4 \pi^{0}$ at SND 

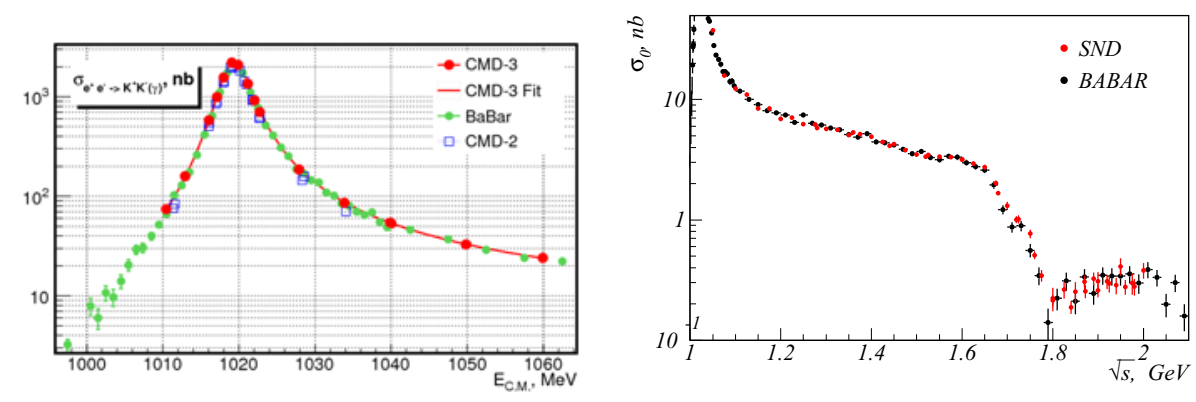

Figure 11. Cross section of $e^{+} e^{-} \rightarrow K^{+} K^{-}$: (left) near the $\phi$ meson at CMD-3, (right) from 1 to $2 \mathrm{GeV}$ at SND

CMD-3 continued studies of various processes with kaons in the final state using good $K / \pi$ separation based on measuring $d E / d x$ in the drift chamber. In Fig. 11 we show the cross section of the process $e^{+} e^{-} \rightarrow K^{+} K^{-}$near the $\phi$ meson at CMD-3 (left) and in the whole energy range at SND (right) [25].

The CMD-3 group has also reported results on the cross section and dynamics of the $K^{+} K^{-} \pi^{+} \pi^{-}$final state, see Fig. 12. While the energy dependence of the cross section is clear and obviously shows the $\phi(1680)$ state, there is still a lot to be done about the dynamics.

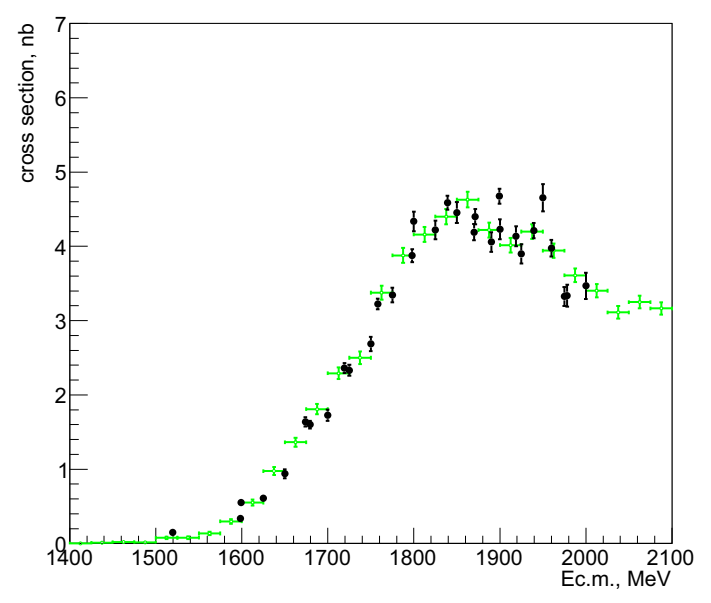

Figure 12. Cross section of $e^{+} e^{-} \rightarrow K^{+} K^{-} \pi^{+} \pi^{-}$at CMD-3 [27].

Using the missing mass method, CMD-3 has also measured the cross section of the process $e^{+} e^{-} \rightarrow K^{+} K^{-} \eta$, Fig. 13. Work is in progress on the process $e^{+} e^{-} \rightarrow$ $K^{+} K^{-} \pi^{0}$.

SND has already published their measurements of the processes with only neutral particles in the final state $e^{+} e^{-} \rightarrow \pi^{0} \pi^{0} \gamma$ [26] and $e^{+} e^{-} \rightarrow \eta \gamma$ [27]. The latter is of particular interest since for the first time events of the process have been found above $1.4 \mathrm{GeV}$, see Fig. 14 .

A study of the nucleon form factors near threshold was continued. SND significantly improved the precision of $\sigma\left(e^{+} e^{-} \rightarrow n \bar{n}\right)$ [28] compared to the previous results from FENICE [29], see Fig. 15.

CMD-3 measured the cross section of the process $e^{+} e^{-} \rightarrow p \bar{p}$ and made an attempt to extract the ratio of

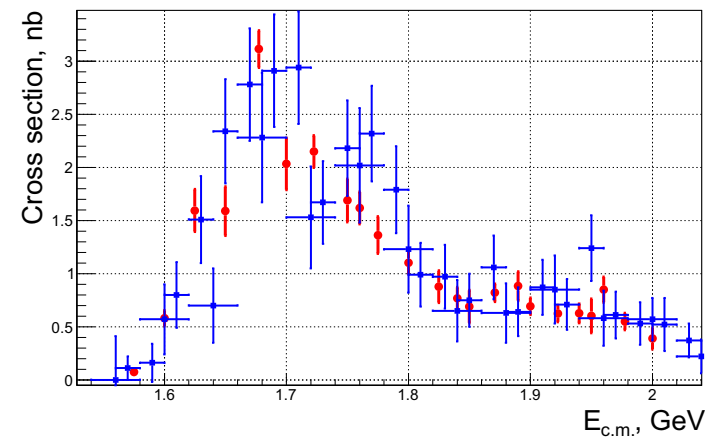

Figure 13. Cross section of $e^{+} e^{-} \rightarrow K^{+} K^{-} \eta$ at CMD-3 [27]

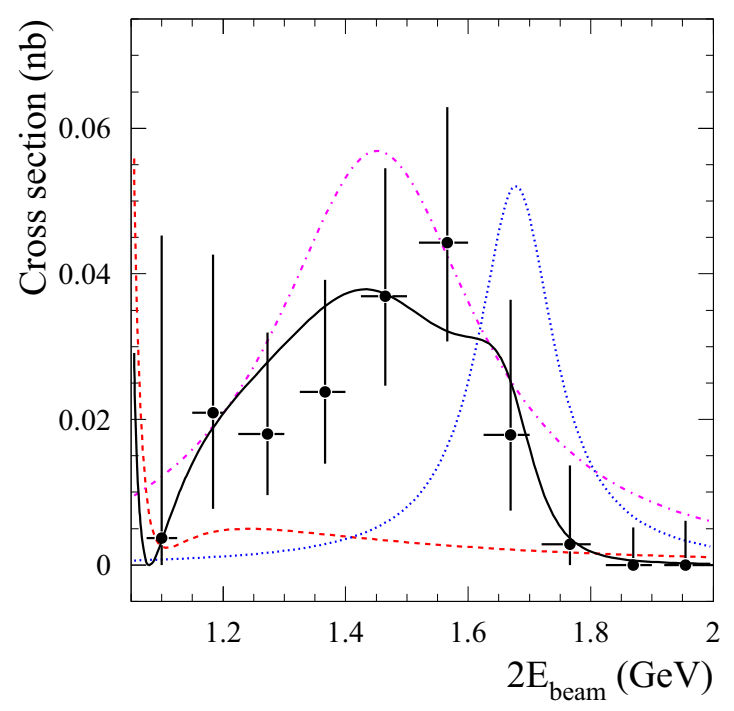

Figure 14. Cross section of $e^{+} e^{-} \rightarrow \eta \gamma$ at SND [27].

the electric and magnetic form factors based on the angular distribution of the final nucleons [30], see Fig. 16.

Both detectors used an original method of Ref. [31] to measure the partial width of a strongly suppressed $\eta^{\prime} \rightarrow$ $e^{+} e^{-}$decay using the inverse process. CMD-3 reported an upper limit of $\Gamma\left(\eta^{\prime} \rightarrow e^{+} e^{-}\right)<0.0024 \mathrm{eV}$ at $90 \% \mathrm{CL}$ based on $2.69 \mathrm{pb}^{-1}$ and one mode of $\eta^{\prime}$ decay [32]. SND used $2.9 \mathrm{pb}^{-1}$ and five modes of $\eta^{\prime}$ decay to improve it to 


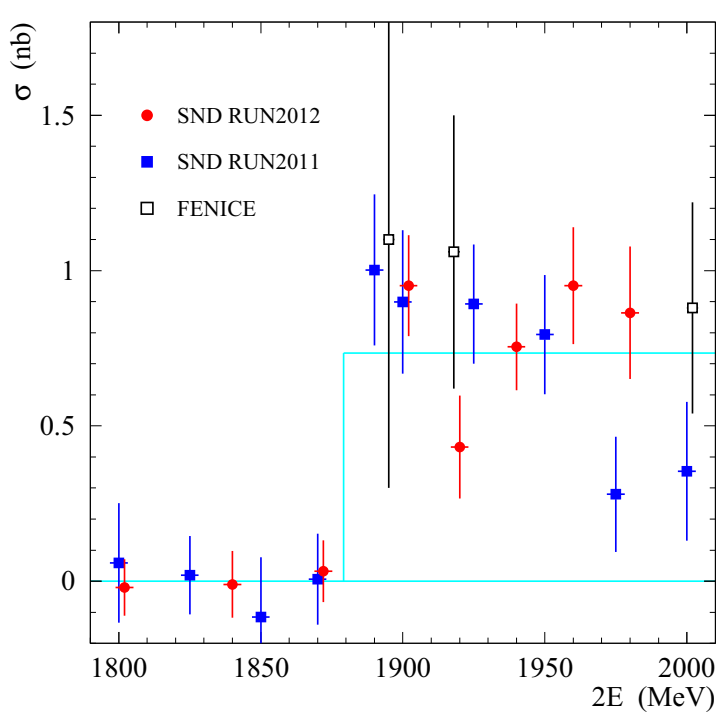

Figure 15. Cross section of the process $e^{+} e^{-} \rightarrow n \bar{n}$ at SND [28]

$<0.0020 \mathrm{eV}$. Finally, they combined the data samples of CMD-3 and SND to find $\Gamma\left(\eta^{\prime} \rightarrow e^{+} e^{-}\right)<0.0011 \mathrm{eV}$ at $90 \%$ CL [33] which is still about two orders of magnitude below the unitary bound. SND has also performed a feasibility study for a search for $\eta \rightarrow e^{+} e^{-}$via $e^{+} e^{-} \rightarrow \eta$ and concluded that the only promising decay mode for that is $\eta \rightarrow 3 \pi^{0}$ [34]. A dedicated two-week run with the luminosity expected at the c.m. energy around the $\eta$ meson mass will allow to improve the existing limit [35].

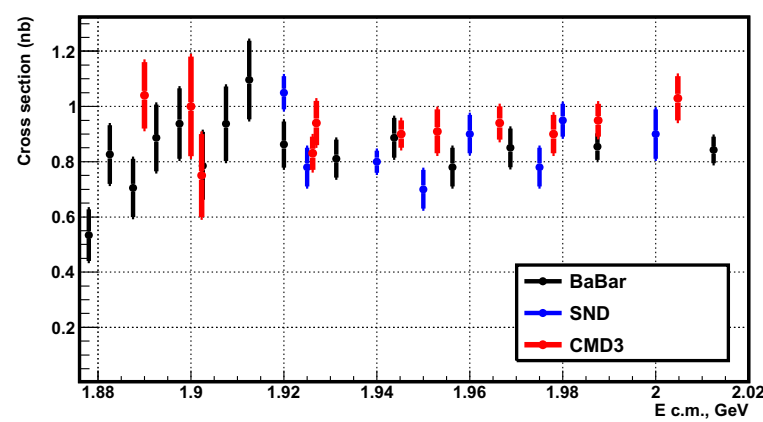

Figure 16. Cross section of the process $e^{+} e^{-} \rightarrow p \bar{p}$ at CMD$3[30]$.

After upgrading the VEPP-2000 collider and commissioning the new injection complex the luminosity of the complex is expected to increase by an order of magnitude. Both detectors will run for another five years with a goal of collecting 1-2 $\mathrm{fb}^{-1}$ and increasing significantly the accuracy of all hadronic channels.

\section{Acknowledgments}

The author thanks G. D'Ambrosio and other Naples colleagues for their excellent organization of the Conference and very kind hospitality. This work was partially supported by the RFBR grants 13-02-00215, 14-02-91332, 15-02-05674 and the DFG grant HA 1457/9-1.

\section{References}

[1] G.W. Bennett et al., Phys. Rev. D 73, 072003 (2006).

[2] M. Davier et al., Eur. Phys. J. C 71, 1515 (2011).

[3] K. Hagiwara et al., J. Phys. G 38, 085003 (2011).

[4] V.P. Druzhinin et al., Rev. Mod. Phys. 83, 1545 (2011).

[5] V.V. Anashin et al. (KEDR Collaboration), arxiv: 1510.02667.

[6] V.V. Anashin et al. (KEDR Collaboration), Phys. Lett. B 703, 543 (2011).

[7] B. Aubert et al. (BaBar Collaboration), Phys. Rev. Lett. 103, 231801 (2009).

[8] J.P. Lees et al. (BaBar Collaboration), Phys. Rev. D 86, 032013 (2012).

[9] F.Ambrosino et al. (KLOE Collaboration), Phys. Lett. B 670, 285 (2009).

[10] F.Ambrosino et al. (KLOE Collaboration), Phys. Lett. B 700, 102 (2011).

[11] D. Babusci et al. (KLOE Collaboration), Phys. Lett. B 720, 336 (2013).

[12] M. Ablikim et al. (BES Collaboration), arXiv:1507.08188.

[13] M.N. Achasov et al. (SND Collaboration), JETP 103, 380 (2006).

[14] R.R. Akhmetshin et al. (CMD-3 Collaboration), Phys. Lett. B 648, 28 (2007).

[15] Yu.M. Shatunov et al., Proc. of EPAC 2000, Vienna, Austria, June 2000, p.439.

[16] M.N. Achasov et al. (CMD-3 and SND Collaborations), Int. J. Mod. Phys. Conf. Ser. 35, 1460388 (2014).

[17] R.R. Akhmetshin et al. (CMD-3 Collaboration), JINST 9, C09003 (2014).

[18] V.M. Aulchenko et al. (SND Collaboration), Phys. Rev. D 91, 052013 (2015).

[19] G.V. Fedotovich et al., Phys. Atom. Nucl. 78, 591 (2015).

[20] V.M. Aulchenko et al. (SND Collaboration), J. Exp. Theor. Phys. 121, 34 (2015).

[21] R.R. Akhmetshin et al. (CMD-3 Collaboration), Phys. Lett. B 723, 82 (2013).

[22] B. Aubert et al. (BaBar Collaboration), Phys. Rev. D 73, 052003 (2006).

[23] J. Haidenbauer et al., Phys. Rev. D 92, 054032 (2015).

[24] P.A.Lukin et al. (CMD-3 Collaboration), Phys. Atom. Nucl. 78, 353 (2015).

[25] E.A. Kozyrev et al. (CMD-3 Collaboration), Phys. Atom. Nucl. 78, 358 (2015).

[26] M.N. Achasov et al. (SND Collaboration), Phys. Rev. D 88, 054013 (2013). 
[27] M.N. Achasov et al. (SND Collaboration), Phys. Rev. D 90, 032002 (2014).

[28] M.N. Achasov et al. (SND Collaboration), Phys. Rev. D 90, 112007 (2014).

[29] A. Antonelli et al. (FENICE Collaboration), Nucl. Phys. B 517, 3 (1998).

[30] R.R. Akhmetshin et al. (CMD-3 Collaboration), Int.J.Mod.Phys.Conf.Ser. 35, 1460457 (2014).

[31] P. Vorobev et al. (ND Collaboration), Sov. J. Nucl. Phys. 48, 273 (1988).
[32] R.R. Akhmetshin et al. (CMD-3 Collaboration), Phys. Lett. B 740, 273 (2015).

[33] M.N. Achasov et al. (SND Collaboration), Phys. Rev. D 91, 092010 (2015).

[34] M.N. Achasov et al. (SND Collaboration), JETP Lett. 102, 266 (2015).

[35] G. Agakishiev et al. (HADES Collaboration), Phys. Lett. B 731, 265 (2014). 